\title{
Administration of Radioactive Iodine Therapy Within 1 Year After Total Thyroidectomy Does Not Affect Vocal Function
}

\author{
Chang Hwan Ryu ${ }^{1}$, Junsun Ryu${ }^{1}$, Youn Mi Ryu ${ }^{1}$, You Jin Lee², Eun-Kyung Lee², Seok-Ki Kim³ ${ }^{3}$ Tae-Sung Kim³, \\ Tae Hyun Kim ${ }^{4}$, Chang Yoon Lee ${ }^{5}$, Seog Yun Park ${ }^{6}$, Ki Wook Chung ${ }^{7}$, and Yuh-S. Jung ${ }^{1}$ \\ ${ }^{I}$ Department of Otolaryngology-Head and Neck Surgery, Center for Thyroid Cancer, Research Institute and Hospital, National \\ Cancer Center, Goyang-si, Gyeonggi-do, Korea; ${ }^{2}$ Department of Internal Medicine, Center for Thyroid Cancer, Research Institute \\ and Hospital, National Cancer Center, Goyang-si, Gyeonggi-do, Korea; ${ }^{3}$ Department of Nuclear Medicine, Center for Thyroid \\ Cancer, Research Institute and Hospital, National Cancer Center, Goyang-si, Gyeonggi-do, Korea; ${ }^{4}$ Department of Radiation \\ Oncology, Center for Thyroid Cancer, Research Institute and Hospital, National Cancer Center, Goyang-si, Gyeonggi-do, Korea; \\ ${ }^{5}$ Department of Radiology, Center for Thyroid Cancer, Research Institute and Hospital, National Cancer Center, Goyang-si, \\ Gyeonggi-do, Korea; ${ }^{6}$ Department of Pathology, Center for Thyroid Cancer, Research Institute and Hospital, National Cancer \\ Center, Goyang-si, Gyeonggi-do, Korea; and ${ }^{7}$ Department of Surgery, Asan Medical Center, University of Ulsan College of Medicine, \\ Seoul, Korea
}

The purpose of this study was to evaluate the impact of radioactive iodine therapy (RIT) on vocal function during the early follow-up period after total thyroidectomy (TT) using perceptive and objective measurements, questionnaires regarding subjective symptoms, and data on vocal function in a prospectively enrolled and serially followed thyroid cancer cohort. Methods: Of 212 patients who underwent $\pi T$ and were screened between January and December 2010 at our hospital, 160 were included in the final analysis. Patients with the following histories were excluded: lateral neck dissection, organic vocal fold disease, external radiotherapy, and voice evaluation during thyroxine withdrawal. Patients were stratified into 3 groups: $\Pi$, $\Pi$ with low-dose RIT (1.1-2.2 GBq), and $\Pi$ with high-dose RIT ( $\geq 3.7 \mathrm{GBq}$ ). Voice evaluations were performed before surgery and at 1, 6, and 12 mo after TT. Results: Vocal characteristics were altered after $\Pi$, including changes on the grade, roughness, and strain scale; increased amplitude perturbation; decreased fundamental frequency; narrowed pitch range; and global disturbances in subjective functional parameters on the voice handicap index. However, the degree of vocal changes among the 3 groups did not significantly differ within the 1-y postoperative follow-up period. According to the results of subgroup analyses of patients who demonstrated good voice outcomes after $\Pi$, there were no significant functional differences among the 3 groups. Conclusion: RIT at any dose does not affect vocal function within $1 \mathrm{y}$ of TT.

Key Words: thyroid cancer; radioactive iodine; voice; total thyroidectomy

J Nucl Med 2015; 56:1480-1486

DOI: 10.2967/jnumed.115.161463

Received May 28, 2015; revision accepted Jul. 24, 2015.

For correspondence or reprints contact: Yuh-S. Jung, Department of Otolaryngology-Head and Neck Surgery, Center for Thyroid Cancer, Research Institute and Hospital, National Cancer Center, 323 Ilsan-ro, Ilsandong-gu, Goyang-si, Gyeonggi-do, 410-769, Republic of Korea.

E-mail: jysorl@ncc.re.kr

Published online Aug. 13, 2015.

COPYRIGHT (C 2015 by the Society of Nuclear Medicine and Molecular Imaging, Inc. hyroid cancer is the most common endocrine malignancy, and its incidence has continuously increased all over the world in recent decades. The most common types of thyroid cancer are papillary $(80 \%)$ and follicular carcinoma (10\%), which demonstrate excellent outcomes and 10-y overall survival rates of $90 \%-$ $95 \%(1,2)$. This is partly attributed to the therapeutic role of adjuvant radioactive iodine (2-9).

Since the first attempt to treat differentiated thyroid carcinoma in 1946, radioactive iodine therapy (RIT) has played an important role in its treatment (10). Radioactive iodine is administered to patients after complete gross surgical resection of differentiated thyroid carcinoma and remnant ablation or adjuvant therapy. Radioactive iodine ablation (or remnant ablation)which refers to destroying residual, microscopically normal thyroid tissue-facilitates the interpretation of serum thyroglobulin levels and the sensitivity of detecting persistent carcinoma on a follow-up whole-body scan $(6,8,9)$. Adjuvant RIT can also eventually decrease the risk of recurrence and mortality (2$5,7,9)$. Many studies support the use of RIT to reduce the rate of recurrence and death (2-9).

The recent American Thyroid Association and National Comprehensive Cancer Network guidelines recommend that most instances of differentiated thyroid carcinoma-except subcentimeter, intrathyroidal carcinoma without regional lymph node metastasisbe considered candidates for RIT after a total thyroidectomy (TT) $(11,12)$. However, despite the apparent benefits of RIT, this treatment may also cause several morbidities, including salivary gland disturbances, depending on the total radioactive iodine dose. Salivary dysfunction may develop earlier within the first year after RIT (13-18).

The voice is one of the most important human functions, and even subtle vocal changes can affect quality of life, particularly among professional voice users $(19,20)$. Salivary dysfunction, which is occasionally induced by RIT, may also closely influence vocal function $(21,22)$. The natural course of radioactive iodine on vocal function after TT has not been systematically studied, and we thus evaluated this in our current study in a prospectively and serially enrolled cohort of thyroid cancer patients (23). Perceptive 
TABLE 1

Baseline Clinical and Vocal Characteristics of Study Population $(n=160)$

\begin{tabular}{|c|c|c|c|c|c|}
\hline Characteristic & Total $(n=160)$ & $\mathrm{TT}(n=54)$ & $\begin{array}{l}\Pi \text { with } 1.1-2.2 \mathrm{GBq} \\
(n=64)\end{array}$ & $\begin{array}{c}\mathrm{TT}+\geq 3.7 \mathrm{GBq} \\
(n=42)\end{array}$ & $P$ \\
\hline Mean age $\pm S D(y)$ & $48.8 \pm 10.4$ & 49. $1 \pm 11.2$ & $47.8 \pm 10.4$ & $49.9 \pm 9.5$ & 0.558 \\
\hline Female $(n)$ & 129 (80.6\%) & $50(92.6 \%)$ & $53(82.9 \%)$ & $26(61.9 \%)$ & 0.001 \\
\hline T stage $(n)$ & & & & & $<0.001$ \\
\hline T1a & $56(35.0 \%)$ & 45 (83.3\%) & $10(15.6 \%)$ & $1(2.4 \%)$ & \\
\hline $\mathrm{T} 1 \mathrm{~b}$ & $10(6.2 \%)$ & $3(5.6 \%)$ & $5(7.8 \%)$ & $2(4.8 \%)$ & \\
\hline $\mathrm{T} 2$ & $3(1.9 \%)$ & 0 & $1(1.6 \%)$ & $2(4.8 \%)$ & \\
\hline T3 & 91 (56.9\%) & $6(11.1 \%)$ & $48(75.0 \%)$ & $37(88.1 \%)$ & \\
\hline$N$ stage $(n)$ & & & & & $<0.001$ \\
\hline No & 102 (63.8\%) & 52 (96.3\%) & $43(67.2 \%)$ & 7 (16.7\%) & \\
\hline $\mathrm{N} 1 \mathrm{a}$ & $58(36.2 \%)$ & $2(3.7 \%)$ & $21(32.8 \%)$ & 35 (83.3\%) & \\
\hline $\begin{array}{l}\text { Mean operation } \\
\text { time } \pm S D \text { (min) }\end{array}$ & $99.7 \pm 28.6$ & $101.5 \pm 23.7$ & $100.6 \pm 34.9$ & $96.05 \pm 23.4$ & 0.620 \\
\hline GRBAS $=0(n)$ & 79 (49.4\%) & 32 (59.3\%) & $24(37.5 \%)$ & $23(54.8 \%)$ & 0.365 \\
\hline \multicolumn{6}{|l|}{ Mean acoustic \pm SD } \\
\hline Jitter (\%) & $1.4 \pm 1.0$ & $1.4 \pm 0.8$ & $1.5 \pm 1.0$ & $1.3 \pm 1.3$ & 0.610 \\
\hline Shimmer (\%) & $4.0 \pm 1.7$ & $3.8 \pm 1.6$ & $4.1 \pm 1.8$ & $4.1 \pm 1.6$ & 0.875 \\
\hline $\mathrm{NHR}(\mathrm{dB})$ & $0.1 \pm 0.3$ & $0.1 \pm 0.0$ & $0.1 \pm 0.3$ & $0.1 \pm 0.0$ & 0.162 \\
\hline $\mathrm{FO}\left(\mathrm{Hz}^{\star}\right)$ & $198.8 \pm 25.8$ & $196.1 \pm 32.5$ & $201.3 \pm 21.1$ & $198.9 \pm 19.6$ & 0.592 \\
\hline \multicolumn{6}{|l|}{ VRP $\left(H z^{*}\right)$} \\
\hline Fmax & $625.6 \pm 210.4$ & $595.7 \pm 199.5$ & $643.6 \pm 222.5$ & $646.6 \pm 206.5$ & 0.441 \\
\hline Fmin & $153.7 \pm 25.0$ & $152.5 \pm 27.2$ & $157.9 \pm 23.1$ & $147.7 \pm 23.8$ & 0.217 \\
\hline VHI & $5.0 \pm 11.4$ & $5.1 \pm 14.0$ & $5.3 \pm 10.3$ & $4.3 \pm 9.3$ & 0.413 \\
\hline
\end{tabular}

and objective measurements and questionnaires designed to yield data on subjective symptoms related to vocal function were used to evaluate this cohort. The results of early and $1-y$ postoperative voice analyses are presented.

\section{MATERIALS AND METHODS}

\section{Patients}

Between January and December 2010, 212 patients who underwent TT for differentiated thyroid carcinoma at our hospital were prospectively screened for this study with the following inclusion criteria: age of $25-80 \mathrm{y}$, history of TT for differentiated thyroid carcinoma, and no organic vocal fold disease such as nodules, polyps, or granulomas. The exclusion criteria included lateral neck metastases and a history of external radiotherapy. Patients were later excluded from the functional analyses if they had postoperative glottic disease, including recurrent laryngeal nerve palsy, or underwent voice analysis during the thyroxine withdrawal period for RIT, because both of these may decrease vocal quality. Eventually, 160 patients were eligible for inclusion in the analyses. All participants provided written informed consent according to the policies and procedures approved by the institutional review board of the National Cancer Center, Korea (NCCNCS-09-294). Patients were stratified according to the received radioactive iodine dose; that is, TT, TT with low-dose RIT (1.1-2.2 GBq), or TT with highdose RIT ( $\geq 3.7 \mathrm{GBq})$.

\section{Treatment}

All surgical procedures were performed by 3 experienced thyroid surgeons, as described previously (23). Prophylactic or therapeutic central neck dissection, which included the pretracheal, prelaryngeal, and bilateral paratracheal nodes, was performed on all patients. RIT was recommended 2-4 mo after surgery. Radioactive iodine was administered as a low-dose (1.1-2.2 GBq 1-2 times per year) or high-dose $(\geq 3.7 \mathrm{GBq})$ treatment, depending on the risk stratifications included in the guidelines of the American Thyroid Association, after a full interdisciplinary discussion (11).

\section{Videostroboscopy}

At the follow-up examinations, videostroboscopy using a $70^{\circ}$ rigid laryngoscope (model RLS 9100 B; Kay Elemetrics) was used to assess impaired vocal fold motility and other organic diseases, including nodules, polyps, and granulomas in the vocal folds.

\section{Voice Evaluation}

All enrolled patients were followed for more than 12 mo. All perceptive and acoustic measurements were performed by a single speech pathologist who did not know the clinical information. Evaluations were performed preoperatively and at 1, 6, and 12 mo after surgery. All patients also reported their answers to the subjective questionnaire at each time point. Perceptive voice quality was assessed using the grade, roughness, breathiness, asthenia, and strain (GRBAS) scale. Acoustic voice analysis was performed using the 
multidimensional voice program (MDVP) and voice range profile (VRP) included with Computerized Speech Lab (model 4150; Kay Elemetrics). The following 4 parameters were selected for MDVP analysis: noise-to-harmonic ratio, short-term frequency perturbation (i.e., jitter [\%]), short-term amplitude perturbation (i.e., shimmer [\%]), and fundamental frequency (F0). Measurements derived from the VRP included the minimal $\left(\mathrm{F}_{\min }\right)$ and maximal $\left(\mathrm{F}_{\max }\right)$ fundamental frequencies. These procedures were repeated 3 times and averaged in each direction. The subjective questionnaire was assessed using the Korean version of the Vocal Handicap Index (VHI), which consists of a 30item questionnaire and includes functional, physical, and emotional subscales. Each item on the VHI is scored on a 5-point scale (range, 0-4), and the highest score is 120 points. On the VHI scales, a higher score indicates a greater perception of functional disability.

\section{Statistical Analysis}

Continuous variables are presented as the mean \pm SD and were compared among groups using 1-way ANOVA. Categoric variables are presented as counts with proportions and were compared among groups using the $\chi^{2}$ test. Changes in voice outcome measures and differences among groups over time were calculated using repeatedmeasures ANOVA. Post hoc testing with the Bonferroni method was performed when appropriate. To compare the F0, $\mathrm{F}_{\max }$, and $\mathrm{F}_{\text {min }}$, only female patients were included in this analysis because of baseline sex-related differences. In this study, $P$ values of less than 0.05 were considered statistically significant. Analyses were performed using STATA/SE, version 10.1 (StataCorp LP).

\section{RESULTS}

\section{Baseline Clinical and Vocal Characteristics}

Our final study cohort consisted of 160 patients with a mean age of $48 \pm 10 \mathrm{y}$. The study series was divided into 3 groups: TT
( $n=54)$, TT with low-dose RIT $(n=64)$, and TT with high-dose RIT $(n=42)$ (Table 1$)$. The median age was similar among groups. Male patients were more common in the group receiving TT with high-dose RIT $(P=0.001)$. Advanced T-stage $(P<0.001)$ and $\mathrm{N}$-stage tumors $(P<0.001)$ were significantly more common in the groups receiving TT with low-dose and high-dose RIT than in the group receiving only TT. However, the operating times among the 3 groups were not different. Before thyroidectomy, all evaluated vocal parameters, including GRBAS scores, acoustic voice parameters (jitter, shimmer, noise-to-harmonic ratio, $\mathrm{F} 0, \mathrm{~F}_{\max }$, and $\mathrm{F}_{\min }$ ), and subjective VHI were similar among all 3 groups. Overall, the baseline functional parameters did not differ among groups.

Among the 160 enrolled subjects, no patient had a thyroxine level of less than $0.89 \mathrm{mg} / \mathrm{mL}$ or a thyroid-stimulating hormone level of more than $4.05 \mu \mathrm{IU} / \mathrm{mL}$ at any voice measurement (before or 1,6 , or 12 mo after TT). This finding suggested that biases in vocal function due to hypothyroidism would be minimal across the follow-up period.

\section{Postoperative Voice Changes After Thyroidectomy}

Analysis after TT revealed significant changes from the baseline values across several GRBAS, MDVP, and VRP parameters, as well as changes in subjective VHI scores; moreover, some of these impairments persisted for up to $12 \mathrm{mo}$. On the GRBAS scale, the overall grade and roughness parameters significantly increased during the $1-y$ postoperative follow-up period $(P<0.001)$. The strain parameter also increased at 1 mo after TT but normalized thereafter (Table 2). Similar to the perceptive scale, the acoustic parameters measured using MDVP and VRP also demonstrated functional impairment. Regarding the MDVP parameters, shimmer and F0 remained significantly impaired through all follow-up

TABLE 2

Vocal Parameters Through Perioperative Course

\begin{tabular}{|c|c|c|c|c|c|}
\hline \multirow[b]{2}{*}{ Parameter } & \multicolumn{4}{|c|}{ Time } & \multirow[b]{2}{*}{ Time } \\
\hline & Pre & $1 \mathrm{mo}$ & $6 \mathrm{mo}$ & $12 \mathrm{mo}$ & \\
\hline \multicolumn{6}{|l|}{ GRBAS } \\
\hline $\mathrm{G}$ & $0.3 \pm 0.5$ & $0.7 \pm 0.7$ & $0.4 \pm 0.5$ & $0.4 \pm 0.5$ & $<0.001$ \\
\hline $\mathrm{R}$ & $0.5 \pm 0.5$ & $0.8 \pm 0.5$ & $0.8 \pm 0.4$ & $0.9 \pm 0.4$ & $<0.001$ \\
\hline B & $0.0 \pm 0.2$ & $0.1 \pm 0.3$ & $0.0 \pm 0.2$ & $0.1 \pm 0.3$ & 0.133 \\
\hline$A$ & 0 & $0.1 \pm 0.2$ & $0.0 \pm 0.1$ & 0 & NA \\
\hline$S$ & $0.0 \pm 0.2$ & $0.3 \pm 0.5$ & $0.1 \pm 0.3$ & $0.1 \pm 0.2$ & $<0.001$ \\
\hline \multicolumn{6}{|l|}{ Acoustic } \\
\hline Jitter (\%) & $1.4 \pm 1.0$ & $1.5 \pm 1.4$ & $1.4 \pm 1.1$ & $1.6 \pm 1.1$ & 0.167 \\
\hline Shimmer (\%) & $4.0 \pm 1.7$ & $4.6 \pm 3.2$ & $3.9 \pm 1.7$ & $4.1 \pm 1.6$ & 0.024 \\
\hline $\mathrm{NHR}(\mathrm{dB})$ & $0.1 \pm 0.3$ & $0.2 \pm 1.3$ & $0.2 \pm 0.5$ & $0.1 \pm 0.0$ & 0.376 \\
\hline $\mathrm{FO}\left(\mathrm{Hz}^{\star}\right)$ & $198.8 \pm 25.8$ & $188.6 \pm 28.6$ & $192.6 \pm 27.9$ & $189.6 \pm 28.6$ & $<0.001$ \\
\hline $\operatorname{Fmax}\left(\mathrm{Hz}^{\star}\right)$ & $625.6 \pm 210.4$ & $433.3 \pm 190.0$ & $528.2 \pm 192.6$ & $557.7 \pm 204.1$ & $<0.001$ \\
\hline Fmin $\left(H z^{*}\right)$ & $153.7 \pm 25.0$ & $146.8 \pm 27.5$ & $158.3 \pm 24.4$ & $158.8 \pm 23.1$ & $<0.001$ \\
\hline \multicolumn{6}{|l|}{ Subjective } \\
\hline VHI & $5.0 \pm 11.4$ & $33.6 \pm 26.2$ & $19.8 \pm 23.6$ & $16.7 \pm 18.5$ & $<0.001$ \\
\hline
\end{tabular}

${ }^{*}$ To compare $\mathrm{F} 0, \mathrm{~F}_{\max }$, and $\mathrm{F}_{\min }$, only female patients were included in this analysis because of baseline sex-related differences. $\mathrm{NA}=$ not applicable; $\mathrm{NHR}=$ noise-to-harmonic ratio. 


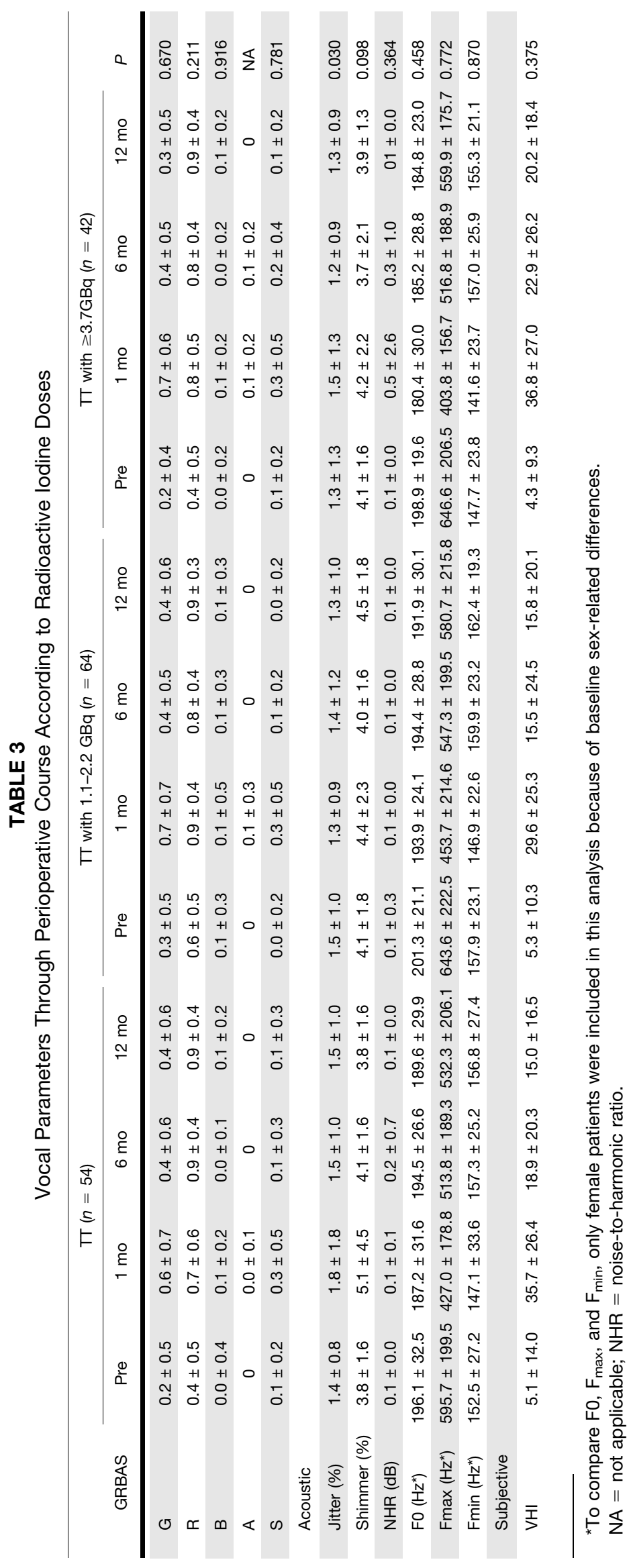

sessions. Meanwhile, jitter and noise-to-harmonic ratio did not demonstrate significant postoperative changes. All VRP parameters, including $\mathrm{F}_{\max }$ and $\mathrm{F}_{\min }$, decreased after TT. According to the subjective questionnaire, VHI demonstrated results that were in agreement with the perceptive and acoustic changes through all follow-up examinations. VHI significantly increased after surgery and persisted through $1 \mathrm{y}$ after surgery.

\section{Effects of RIT on Vocal Quality After TT}

The RIT dose had no significant effect on vocal function during the treatment course. At all time points there were consistently no significant differences among the 3 groups in terms of GRBAS, acoustic parameters, or subjective VHI scores (Table 3). Because this lack of significant functional differences among groups could be due to bias and the functional consequence of the surgery itself, we further analyzed subgroups that were divided by vocal status at 1 mo after TT (i.e., the time point before RIT) with the expectation that this would allow us to better focus on the unbiased effects of RIT on vocal function. Thus, we divided the subgroups by their immediate postoperative vocal outcomes using 3 vocal parameters. The group with good voice outcomes was defined using the following criteria based on modification of a previous study: in terms of the perceptive scale, a less than 2 point increase on GRBAS from baseline ( $\triangle$ GRBAS); in terms of acoustic parameters, less than 2 acoustic parameters demonstrating significant changes of $11 \%$ or more from baseline ( $\Delta$ acoustic); and in terms of the subjective questionnaire definition, fewer than 20 changes in VHI from baseline ( $\Delta \mathrm{VHI})(24)$.

This subgroup analysis also showed that there were no differences in vocal quality among the 3 treatment groups over time within the group with good voice outcomes. There were no significant differences in terms of grade, roughness, or strain (Fig. 1) or in terms of $F_{\max }, F_{\min }, F 0$, or shimmer (Fig. 2) in patients with good voice outcomes, according to the $\Delta$ GRBAS and $\Delta$ acoustic parameters $(\geq 11 \%)$, respectively. The VHI results were in line with the GRBAS and acoustic parameters. There were no differences among the 3 groups in patients with good voice outcomes, as defined by $\Delta \mathrm{VHI}$, throughout the study period (Fig. 3).

\section{DISCUSSION}

Although administration of less treatment for thyroid cancer may affect vocal function less, most studies evaluate the functional effects of surgical treatment, and as we previously reported, the surgical extent affects vocal outcomes after thyroidectomy (23). Vocal impairments could be due to an injured recurrent or superior laryngeal nerve, and vocal fold edema could be due to excessive tracheal traction, disrupted laryngeal venous drainage, dysfunctional strap muscles, or laryngotracheal fixation after the surgery (25-28). On the other hand, little is known about the functional consequences of RIT, particularly in terms of vocal function. In our current study, we focused on the exclusive effects of RIT on vocal function in a controlled cohort, and we tried to maximally exclude other biases such as the 


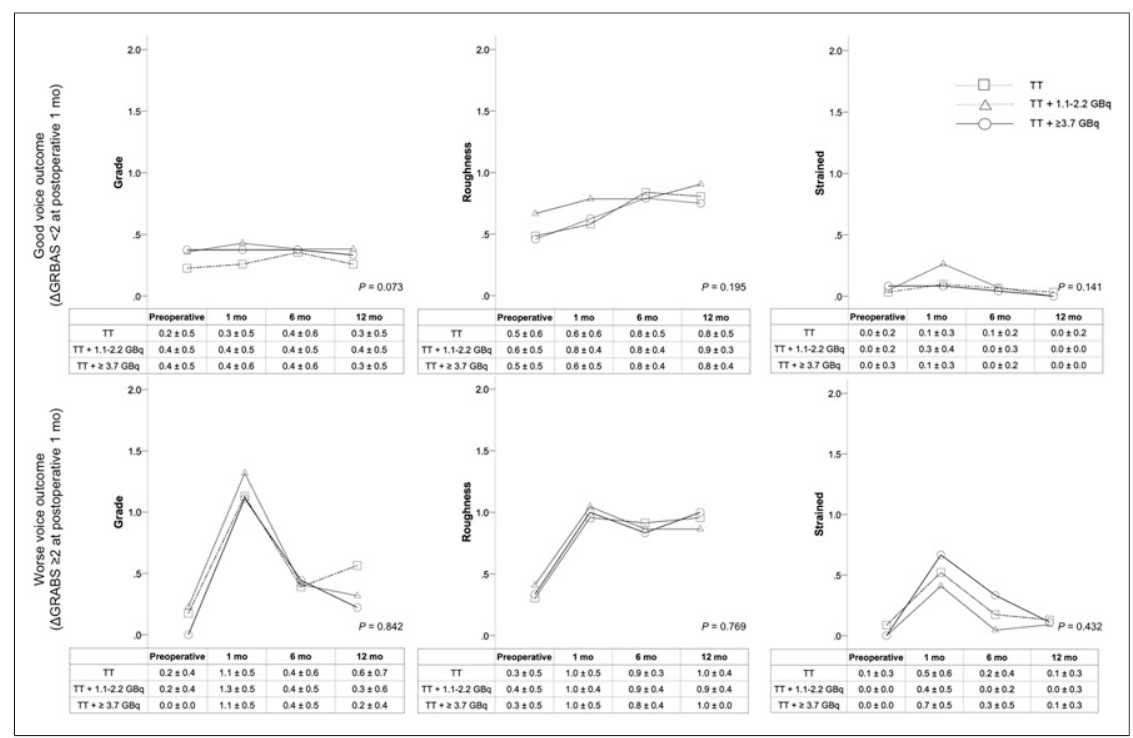

FIGURE 1. Serial patterns of typical functional parameters on perceptive measurements according to radioactive iodine doses after $\Pi T$ stratified by immediate postoperative voice outcomes (1 mo after surgery).

effects of surgical extent (23). Because the indications and doses for RIT are now being reestablished for revised guidelines on differentiated thyroid cancer, these data could more precisely predict the functional consequences of contemporary treatment strategies.

To minimize possible biases that result from differences in surgical extent, only patients who underwent TT were included in our current analysis. Moreover, we excluded patients if they had postoperative glottic diseases such as vocal cord palsy, because these can decrease vocal quality. We also excluded patients who underwent voice evaluations during the thyroxine withdrawal period because an insufficient thyroid hormone status may decrease vocal quality by causing edema of the subepithelial space (29). Our data indicate that almost all of the 160 prospectively enrolled patients who underwent TT presented with vocal problems and that the vocal qualities of these patients remained impaired even at 12 mo after TT (23-27). However, our study findings also consistently show that additional RIT did not affect vocal quality, at least during the 1-y follow-up period, and did not demonstrate any dose-dependent effects of radioactive iodine on vocal functions. Serial GRBAS evaluations throughout the study revealed a worsening in the grade, roughness, and strain parameters, but these differences were not significantly different among our 3 RIT study groups. The acoustic parameters also demonstrated similar changes in our study subjects. The $F_{\max }$ and $F_{\min }$ values of the VRP parameters remained impaired after surgery, but there were no functional differences at any time point. Of the MDVP parameters, F0 and shimmer (which reflect voice changes after surgery) did not demonstrate differences among the 3 groups either. The subjective VHI scale agreed with the perceptive and objective parameters. Global VHI disturbance was observed during the postoperative follow-up period, but this impairment was not affected by the RIT dose.

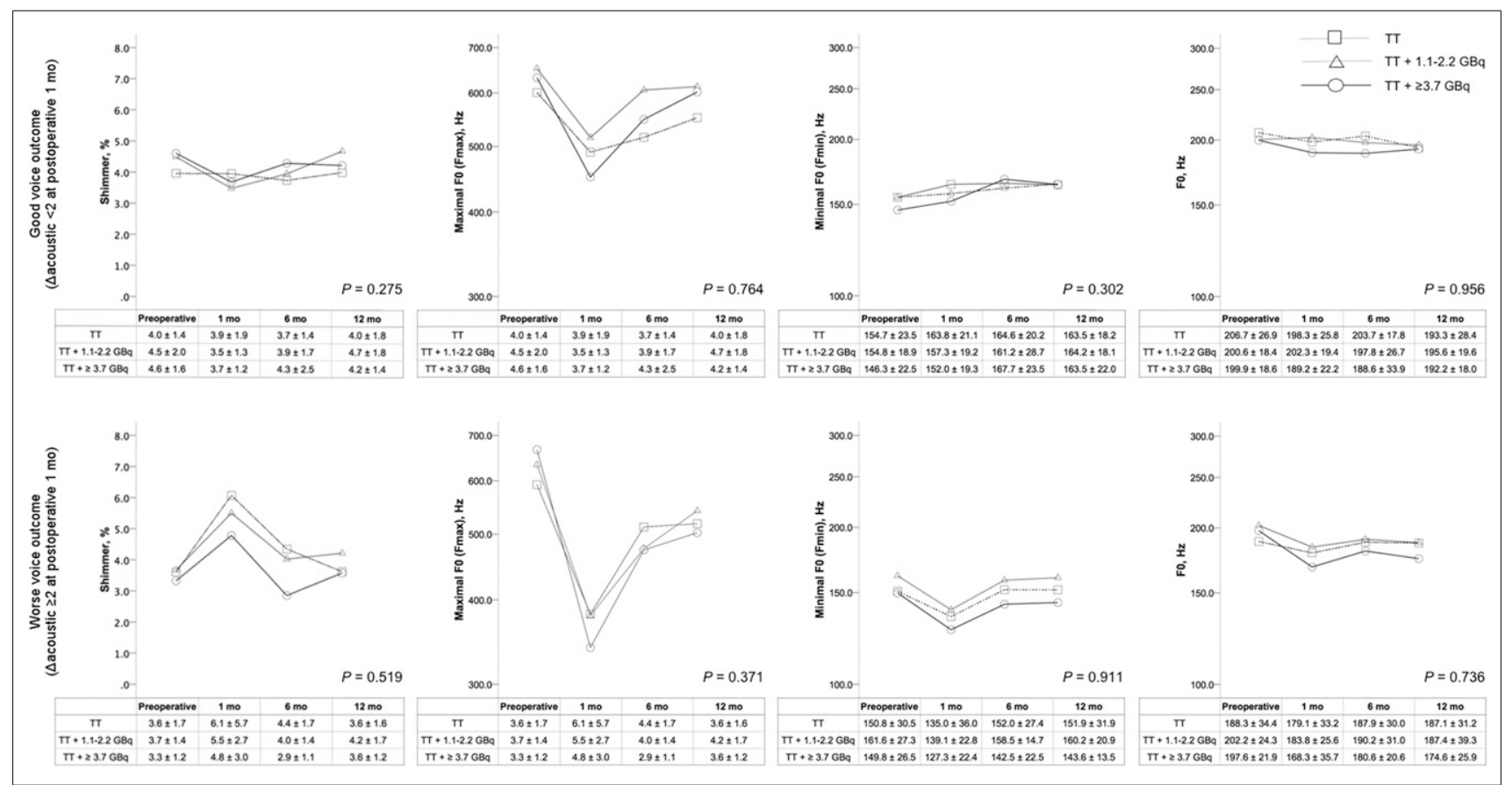

FIGURE 2. Serial patterns of typical functional parameters on acoustic measurements according to radioactive iodine doses after $T T$ stratified by immediate postoperative voice outcomes (1 mo after surgery). 


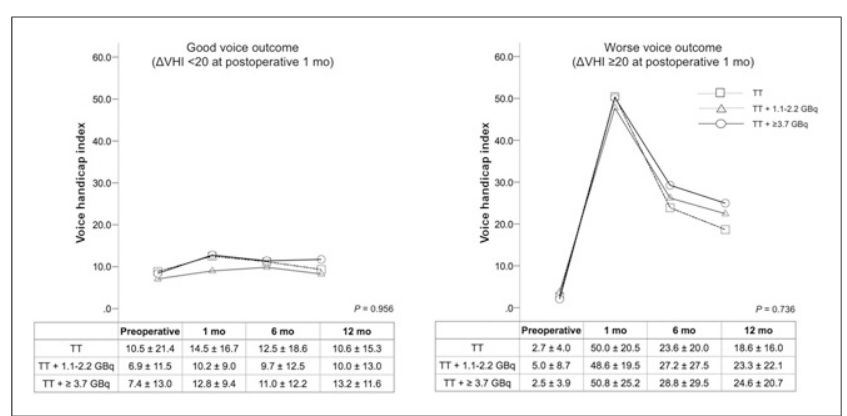

FIGURE 3. Serial patterns of typical functional parameters on subjective questionnaire measurements $(\mathrm{VHI})$ according to radioactive iodine doses after $\Pi$ stratified by immediate postoperative voice outcomes (1 mo after surgery).

To minimize surgery-driven biases and focus on the unbiased functional effects of RIT, we further analyzed subgroups that were divided by vocal status at 1 mo after TT, which is the time point before RIT. We assumed that the impact of surgery on vocal quality would be significant enough to mask the additional effects of RIT. In this step, we used postoperative 1-mo vocal status as the standard for comparison (24). Of note, this subgroup analysis also revealed that RIT did not affect vocal functions through $1 \mathrm{y}$ after TT. By any definition of good voice outcomes, comparison of the 3 groups classified according to RIT dose did not reveal any functional differences. This finding is in line with a previous study on the impact of RIT on vocal quality in patients with Graves disease (30). Recent studies also report no statistical differences in hoarseness associated with RIT dose $(31,32)$. Our present data consistently indicate that RIT has little functional impact in immediate postoperative settings, even on comprehensive voice evaluations that assess perceptive, acoustic, and subjective parameters. However, this finding should be interpreted with caution because salivary gland dysfunction may progress over time after RIT (13-18). Theoretically, RIT may increase the risk of dysphonia because it can affect salivary function. RIT can induce obstructive sialadenitis and, consequently, salivary gland dysfunction. Salivary dysfunction after RIT is reportedly highly prevalent (15-18). Altered salivary flow may affect vocal function because the hydration status of the larynx can affect vocal function and phonatory efforts $(21,22,33-35)$. Because RIT-induced xerostomia usually develops $1 \mathrm{y}$ after RIT, it is possible that the longterm effects of RIT on the voice may differ at this time point. Given these notions, long-term follow-up studies on our current cohort are needed.

Despite the limitations of our current data, our study still provides comprehensive evidence of the slight impact of RIT on vocal function within $1 \mathrm{y}$ after TT. Most voice impairments occurred during the early postoperative follow-up period, and the major determinant of vocal quality was surgery-driven early vocal status (29). RIT did not affect vocal function in a dose-dependent manner. Patients with good voice outcomes at 1 mo after surgery mostly maintained their vocal quality, which was not worsened by RIT. Hence, meticulous functional surgery is the major determinant of optimal vocal outcomes, and RIT has little additional impact. Further studies on the long-term impact of voice impairment due to sialadenitis-related xerostomia are warranted to validate and expand our current results.

\section{CONCLUSION}

Although vocal characteristics were altered after thyroidectomy, further addition of radioactive iodine at any dose within $1 \mathrm{y}$ after surgery had little impact on vocal function. Because RIT may have been thought of as being detrimental to phonation, our findings may lessen concern about its administration after thyroidectomy.

\section{DISCLOSURE}

The costs of publication of this article were defrayed in part by the payment of page charges. Therefore, and solely to indicate this fact, this article is hereby marked "advertisement" in accordance with 18 USC section 1734 . No potential conflict of interest relevant to this article was reported.

\section{REFERENCES}

1. Siegel R, Naishadham D, Jemal A. Cancer statistics, 2013. CA Cancer J Clin. 2013;63:11-30.

2. Kim WB. A closer look at papillary thyroid carcinoma. Endocrinol Metab (Seoul). 2015;30:1-6.

3. Samaan NA, Schultz PN, Hickey RC, et al. The results of various modalities of treatment of well differentiated thyroid carcinomas: a retrospective review of 1599 patients. J Clin Endocrinol Metab. 1992;75:714-720.

4. Mazzaferri EL, Jhiang SM. Long-term impact of initial surgical and medical therapy on papillary and follicular thyroid cancer. Am J Med. 1994;97:418428.

5. Mazzaferri EL, Jhiang SM. Differentiated thyroid cancer long-term impact of initial therapy. Trans Am Clin Climatol Assoc. 1995;106:151-168.

6. Mazzaferri EL. Thyroid remnant ${ }^{131} \mathrm{I}$ ablation for papillary and follicular thyroid carcinoma. Thyroid. 1997;7:265-271.

7. Taylor T, Specker B, Robbins J, et al. Outcome after treatment of high-risk papillary and non-Hürthle-cell follicular thyroid carcinoma. Ann Intern Med. 1998;129:622-627.

8. Sawka AM, Brierley JD, Tsang RW, et al. An updated systematic review and commentary examining the effectiveness of radioactive iodine remnant ablation in well-differentiated thyroid cancer. Endocrinol Metab Clin North Am. 2008;37: $457-480$.

9. Van Nostrand D. The benefits and risks of I-131 therapy in patients with welldifferentiated thyroid cancer. Thyroid. 2009;19:1381-1391.

10. Seidlin SM, Marinelli LD, Oshry E. Radioactive iodine therapy; effect on functioning metastases of adenocarcinoma of the thyroid. J Am Med Assoc. 1946;132: 838-847.

11. Cooper DS, Doherty GM, Haugen BR, et al. Revised American Thyroid Association management guidelines for patients with thyroid nodules and differentiated thyroid cancer. Thyroid. 2009;19:1167-1214.

12. Evidence-based cancer guidelines, oncology drug compendium, and oncology continuing medical education. National Comprehensive Cancer Network website. http://www.nccn.org/. Accessed August 12, 2015.

13. Malpani BL, Samuel AM, Ray S. Quantification of salivary gland function in thyroid cancer patients treated with radioiodine. Int J Radiat Oncol Biol Phys. 1996;35:535-540.

14. Mandel SJ, Mandel L. Radioactive iodine and the salivary glands. Thyroid. 2003;13:265-271.

15. Solans R, Bosch J-A, Galofré P, et al. Salivary and lacrimal gland dysfunction (Sicca syndrome) after radioiodine therapy. J Nucl Med. 2001;42:738743.

16. Jeong SY, Kim HW, Lee S-W, Ahn B-C, Lee J. Salivary gland function 5 years after radioactive iodine ablation in patients with differentiated thyroid cancer: direct comparison of pre- and postablation scintigraphies and their relation to xerostomia symptoms. Thyroid. 2013;23:609-616.

17. Caglar M, Tuncel M, Alpar R. Scintigraphic evaluation of salivary gland dysfunction in patients with thyroid cancer after radioiodine treatment. Clin Nucl Med. 2002;27:767-771.

18. Raza H, Khan AU, Hameed A, Khan A. Quantitative evaluation of salivary gland dysfunction after radioiodine therapy using salivary gland scintigraphy. Nucl Med Commun. 2006;27:495-499.

19. Roy N, Merrill RM, Gray SD, Smith EM. Voice disorders in the general population: prevalence, risk factors, and occupational impact. Laryngoscope. 2005;115: 1988-1995. 
20. Mirza N, Ruiz C, Baum ED, Staab JP. The prevalence of major psychiatric pathologies in patients with voice disorders. Ear Nose Throat J. 2003;82:808$810,812,814$.

21. Roh J-L, Kim A-Y, Cho MJ. Xerostomia following radiotherapy of the head and neck affects vocal function. J Clin Oncol. 2005;23:3016-3023.

22. Roh J-L, Kim HS, Kim A-Y. The effect of acute xerostomia on vocal function. Arch Otolaryngol Head Neck Surg. 2006;132:542-546.

23. Ryu J, Ryu YM, Jung Y-S, et al. Extent of thyroidectomy affects vocal and throat functions: a prospective observational study of lobectomy versus total thyroidectomy. Surgery. 2013;154:611-620.

24. Vicente DA, Solomon NP, Avital I, et al. Voice outcomes after total thyroidectomy, partial thyroidectomy, or non-neck surgery using a prospective multifactorial assessment. J Am Coll Surg. 2014;219:152-163.

25. Sinagra DL, Montesinos MR, Tacchi VA, et al. Voice changes after thyroidectomy without recurrent laryngeal nerve injury. J Am Coll Surg. 2004;199:556-560.

26. Stojadinovic A, Shaha AR, Orlikoff RF, et al. Prospective functional voice assessment in patients undergoing thyroid surgery. Ann Surg. 2002;236:823-832.

27. Hong KH, Kim YK. Phonatory characteristics of patients undergoing thyroidectomy without laryngeal nerve injury. Otolaryngol Head Neck Surg. 1997;117: 399-404.
28. Aluffi P, Policarpo M, Cherovac C, Olina M, Dosdegani R, Pia F. Postthyroidectomy superior laryngeal nerve injury. Eur Arch Otorhinolaryngol. 2001;258:451-454.

29. Ritter FN. The effect of hypothyroidism on the larynx of the rat: an explanation for hoarseness associated with hypothyroidism in the human. Ann Otol Rhinol Laryngol. 1964;73:404-416.

30. Isolan-Cury RW, Monte O, Cury AN, et al. Acute effects of radioiodine therapy on the voice and larynx of Basedow-Graves patients. Braz J Otorhinolaryngol. 2008;74:224-229.

31. Schlumberger M, Catargi B, Borget I, et al. Strategies of radioiodine ablation in patients with low-risk thyroid cancer. $N$ Engl J Med. 2012;366:1663-1673.

32. Mallick U, Harmer C, Yap B, et al. Ablation with low-dose radioiodine and thyrotropin alfa in thyroid cancer. $N$ Engl J Med. 2012;366:1674-1685.

33. Leydon C, Sivasankar M, Falciglia DL, Atkins C, Fisher KV. Vocal fold surface hydration: a review. $J$ Voice. 2009;23:658-665.

34. Fisher KV, Ligon J, Sobecks JL, Roxe DM. Phonatory effects of body fluid removal. J Speech Lang Hear Res. 2001;44:354-367.

35. Jiang J, Verdolini K, Aquino B, Ng J, Hanson D. Effects of dehydration on phonation in excised canine larynges. Ann Otol Rhinol Laryngol. 2000;109: $568-575$. 